\title{
Study on the applicability of turbulence model in the simulation of vortex
}

\section{in pump sump}

\author{
Hua Chen
}

Hubei University of Science and Technology

\begin{abstract}
Key words: turbulence model; pump sump; Vortex simulation; Application research
\end{abstract}
Abstract: In the pumping station engineering system, the inlet sump and inlet channels are very important components. Surface vortex, sidewall attached vortex and floor attached vortex of the pump sump is one of the important factors affecting the stable operation of the pump. In order to represent the influence of the vortex in the pump sump better, can construct the applicability model, model $\mathrm{k}-\varepsilon$, model RNG $\mathrm{k}-\varepsilon$,model Realizable $\mathrm{k}-\varepsilon$. Using these three different turbulence models to simulat and numerical calculate the inlet flow field can get more information and the related parameters about the position and intensity of vortex in the pump sump, to further comparative analysis of the forecasting result in different models. Through simulation analysis, the Realizable k- $\varepsilon$ model predicts the locations and vorticity of free surface vortex, sidewall attached vortex and floor attached vortex more accurately than the other models, and the prediction of the tangent velocity and the circular rector is more consistent with the test value, which can provide reliable basis for the design and optimization of the pump sump.

Vortex will produce in the process of internal flow in the open station, and these vortex will have an impact on the state of the pump inlet

Vortex pump station into the pool will be in the open type occurring in the process, the internal flow and produces the vortex will affect state at the inlet of the pump, affect the pump running, even lead to pump cavitation and vibration and noise in physical phenomena such as increased, thereby reducing the stability and security of the normal operation of the pump. At present, there is no open type pump into the pond and reliable design reference, according to the conventional model test technology or design modification, and it is usually need to spend more money and time. Under the constant development and progress of science and technology, computational fluid dynamics by using less money, less time, higher efficiency, higher cost advantage. At present, most of them adopt when Renault are method (RANS) with both sides cheng turbulence model split open type pump station into the pool of internal flow numerical simulation, but the simulation effect is not significant. As a result, some researchers put forward a new method, namely the application model to carry on the numerical simulation, can be more in-depth research on vortex ${ }^{[1]}$. Later, some researchers are compared with those of the model applicability of the model, found similar results, have been the great influence of turbulence model, the model of maximum vortex at the center of the vortex strength did not make an accurate assessment, and the researchers compared with the RNG model, further within the pool on vortex intensity to predict the effect ${ }^{[2]}$. In addition, the researchers to improve the RNG model and Realizable model, further widen the application field, but there is no research on numerical model to compare the applicability in open type into the pool and analysis ${ }^{[3]}$. Here, this article will be to the model, the Realizable model, the RNG model comparison and analysis, in order to obtain more comprehensive vortex position and intensity information. 


\section{Calculation model and turbulent model}

Generally speaking, the flow into the pool is incompressible flow, the continuity equation can be used in this process, namely:

$$
\frac{\partial \overline{\mathbf{u}}_{i}}{\partial x_{i}}=0
$$

According to the eddy viscosity hypothesis, Reynolds stress and the average velocity relationship can be established, in the equation, the continuity equation and momentum equation of equation in closed form. The current model or RNC, Realizable model for turbulent variables and, among them, the assumption of turbulent viscosity of scalar, is both the most basic of the cheng turbulence model; Model based on the RNC, also on the rotation and the influence of the rotational flow, can ensure the hydrocyclone with a curved wall flow effect is better, higher adaptability. Realizable model does not need to assume that $\mathrm{Cu}$ coefficient, can establish the two-way connection with strain rate, thus the model under the condition of strain rate resulting in a decrease of normal stress ${ }^{[4]}$.

Researchers have conducted the PIV pumping station into the pool model, this model as a research reference samples, the model of pipe diameter for $=78 \mathrm{~mm}$, outside diameter $=98$, the center of the suction pipe for the origin of coordinates of the model. In order to can reduce the influence of boundary conditions on the simulation, can lengthen slightly on exports, computational domain division method applied to unstructured network, but given that the flow of the suction pipe accessories change has certain complexity, therefore all the more to the grid on the encryption process, the final total control of the grid node in 824501.

As long as the import conditions and adapt to meet all the indicators for imported speed, speed and direction can be identified; Applied to the second category of homogeneous boundary condition as a condition of export, don't need to wave on the surface of the additional consideration, only need to the plane of symmetry can be simplified. Above, embodies the principle of free surface treatment according to the method in the study of multiple application effect markedly in [5]. The finite volume method of discrete control equation and second order central difference grid method is used to set diffusion, and second-order windward format setting flow, using SIMPLEC algorithm for pressure and velocity coupling.

\section{The results and analysis}

To simulate the bin pump station into the pool of three-dimensional turbulent flow numerical, found the attached bottom vortex flow and the characteristics of the intensity distribution, use, said, the strength of the vortex, the inner diameter of the suction pipe. Different model calculation results are obtained, and each model will vortex flow, speed, moderate intensity distribution of the vortex and show it. Through observation, can be found in three models on the bottom of the attached vortex position are calculated, the results showed that position be side suction center, three models only Realizable model close to actual vortex forms, and swirl strength calculation is larger than other two kinds of model calculation results. Can also see that in the left side of the pool, on the right side wall and attached to the wall is also evident in the vortex distribution characteristics, among them, the left side of the size and the form and attached to the wall of vortex is RNG model and the model test of large difference, only a Realizable model predicts vortex form and close to the size and the experiment, further shows the authenticity and reliability of the Realizable model [6]. By looking at the right side of the vortex simulation results can be found attached to the wall, and the position and test results of predicting vortex is RNG model of large difference, and Realizable model and 
prediction model and the vortex position has a slight deviation, and look from the position of the vortex line concentration, and the test result is more close to the Realizable model. Through the observation after wall coanda vortex can learn, look from the test results, and upper part is the main part of the vortex, three models have a consistent prediction results: there are two attached to the wall of vortex. Among them, forecast the vortex position deviation is still RNG model with the model, although the Realizable model on the under side of the main vortex, but top still has great swirl intensity, while the upper position with the test result is no larger deviation. Can also be observed in the experiment into the pool surface and surface vortex line condition and intensity distribution. Look from the position of vortex, and the predicted value is more close to Realizable model, and the three models have said spiral shape is round, all with the actual test measured flat circular vortex forms no larger deviation, RNG model predicting vortex intensity is bigger.

Maximize the intensity of vortex is the center, based on integral swirl intensity range, average flow velocity can be get the suction inlet cross section. By analyzing the different position of vortex tangential velocity and circulation distribution can be found that on average, Realizable model predicts the tangential velocity, compared with other two kind of model is more close to overestimate the tangential velocity distribution is the RNG model, the model is underestimated the tangential velocity value, forecasting curve trend with the test result is more close to. On the circulation distribution, three kinds of model and the prediction result is close to, only model and forecast deviation slightly larger, there is no obvious difference. For vortex left attached to the wall, is the RNG model from the point of view of the tangential velocity distribution with the test closer, followed by Realizable model and the model [7]. From the trend of the curve, the predicted results with the test is more close to the Realizable model with the model. Attached to the wall on the right side of vortex on the tangential velocity and circulation distribution prediction is RNG model of above test, but the closer to the model and the Realizable model test. Three models on the back wall vortex tangential velocity distribution trend forecasting, failed to obtain significant effect, but overall the RNG model and Realizable model prediction and experimental closer. Three turbulence models for predicting surface vortex, the tangential velocity prediction are overvalued, on the trend of numerical and experimental is relatively close to the model and the Realizable model.

\section{Conclusion}

It can predict with bottom vortex and with wall vortex position is Realizable model and standard model. By comparison, with the test have obvious deviation RNG model; Look from the spiral shape prediction results, Realizable model, compared with other two kinds of model and the actual closer; Look from the vortex tangential velocity and circulation, Realizable model closer with the test results, and on the left side of the attached to the wall of vortex forecasting more accurate. To sum up, Realizable model compared with other two kinds of turbulence model has a more accurate prediction precision, into the pool is open type pumping station internal vortex flow numerical simulation is more ideal choice.

\section{Reference}

[1], liu chao, ji-ren zhou, etc. Based on two-way RNG turbulent model pump station outlet conduit flow calculation [J]. Advances in water science, 2010, 15 (1) : 109-112.

[2] CongGuoHui fu-jun wang. The applicability of the turbulence model in pumping station into the pool of vortex study [J]. Journal of agricultural engineering, 2010, 24 (6) : 31-35.

[3] CongGuoHui fu-jun wang. The applicability of the turbulence model in pumping station into the 
pool of vortex simulation research [C] / / 2010 national large pumping station renovation for discussion and exchange new technology, new products meeting proceedings. 2010:318-324.

[4], liu chao, following the equal. Based on the RNG turbulent model of vertical axial-flow pump station three-dimensional flow numerical simulation and performance prediction [J]. Journal of mechanical engineering, 2010, (3) : 252-257.

[5], liu chao, Xue Jian etc. Based on the CFD analysis of flow pumping station of elbow inlet flow hydraulic characteristics research [J]. Journal of applied basic and engineering science journal, 2010 (6) : 891-899.

[6], liu chao, ji-ren zhou, etc. The bottom of the water pump station candy two-dimensional numerical simulation of turbulence flow around [J]. Journal of agricultural machinery, 2010, 4 (3) : 37-39. 